\section{RISK FACTORS ASSOCIATED WITH LIFE THREATENING INFECTIONS IN CHILDREN WITH FEBRILE NEUTROPENIA A DATA MINING APPROACH}

doi:10.1136/archdischild-2012-302724.0851

'Z Badiei, ${ }^{2} \mathrm{MH}$ Alami, ${ }^{3} \mathrm{M}$ Khalesi, ${ }^{1} \mathrm{H}$ Farhangi, ${ }^{1} \mathrm{~A}$ Banihashem, ${ }^{4} \mathrm{AR}$ Razavi, ${ }^{5} \mathrm{HR}$ Kianifar, 'A Ghasemi. 'Pediatric Hematology-Oncology, Dr Shaikh Pediatric Hospital, Mashhad University of Medical Sciences; ${ }^{2}$ Pediatric Infectious Disease, Emam Reza Hospital, Mashhad University of Medical Sciences; '3Pediatric, Dr Shaikh Pediatric Hospital, Mashhad University of Medical Sciences; ${ }^{4}$ Department of Biomedical Engineering, Division of Medical Informatics, Mashhad University of Medical Sciences; ${ }^{5}$ Pediatric Gastroentrology, Ghaem Hospital, Mashhad University of Medical Sciences, Mashhad, Iran

Purpose To determine risk factors(RF) and their relationship with life threatening infection (LTI) in children with febrile neutropenia (FN).

Method In this cross-sectional study, from December 2008 to November 2009, all children with FN admitted to Dr Sheikh Pediatric Hospital were enrolled. For each patient, demographic, clinical and laboratory data were recorded and they were followed up for occurrence of LTI.

Results 120 episodes of FN in 68 patients were analyzed. The most common underlying disease was Acute Lymphoblastic Leukemia (ALL) (53.3\%), 9 (7.5\%) died from an infection and 35 patients (29.1\%) had a LTI. Five variables were identified as risk factors for LTI i.e. body temperature $\geq 39 c^{\circ}(p=0.000)$, presence of mucositis $(\mathrm{p}=0.000)$, abnormal chest $\mathrm{x}$-ray $(\mathrm{p}=0.001)$, platelet count less than $20000 / \mathrm{mm}^{3}(p=0.000)$ and absolute neutrophil count less than 100/ $\mathrm{mm}^{3}(\mathrm{p}=0.001)$.

Risk of LTI was increasing according to number of RFs presented at the beginning of admission (from $2.8 \%$ in patients without RF to $100 \%$ in patients with $5 \mathrm{RF}$ ).

Data mining analysis showed relationship between risk factors with platelet count as the most important variable in the high risk group for LTI.

Conclusion Evaluation of important RFs and judging the severity of patients' condition by studying the importance and relationship between RF at the time of admission can be a useful method for screening LTI in children with FN.

\section{RISK FACTORS FOR CORONARY ARTERY LESIONS IN KAWASAKI DISEASE}

doi:10.1136/archdischild-2012-302724.0852

'A Azhar, ${ }^{2}$ A Al-Attas. 'Pediatrics; ${ }^{2}$ King Abdulaziz University, Jeddah, Saudi Arabia

Introduction Kawasaki disease (KD) is an acute self-limited vasculitis that can become fatal if left untreated. The aim of this study was to identify the risk factors associated with the development of coronary artery lesions (CAL) in pediatric patients with KD.

Methods We performed a retrospective chart review of pediatric patients diagnosed with KD who were admitted to King Abdulaziz University Hospital, Jeddah between January 2001 and December 2011. Descriptive statistics was performed using the Statistical Package for the Social Sciences.

Results Forty-four patients were diagnosed with $\mathrm{KD}$. There were $27(61.4 \%)$ boys and 17 (38.6\%) girls. The mean age of the patients was 26.7 months (range 1.5-108 months). Twenty-three patients (52.3\%) had complete Kawasaki, while $21(47.7 \%)$ had incomplete $\mathrm{KD}$. CAL were found in 16 patients $(61.5 \%)$ with incomplete $\mathrm{KD}$ and in $10(38.5 \%)$ with complete Kawasaki ( $p=0.05)$. CAL were more frequent in males $(p=0.045)$, in the $1-5$ year age group $(p=0.045)$, in children with fever of more than 5 days $(p=0.01)$ and in children who received intravenous immunoglobulin (IVIG) 10 days after the onset of fever ( $\mathrm{p}=0.03$ ). There was no significant relationship between CAL and nationality and other clinical systemic manifistation. There was no relationship between laboratory findings and the development of CAL before and after IVIG administration.

Conclusion CAL are more frequent in patients with incomplete KD. Risk factors for CAL are age between 1-5 years, male gender, and fever of $>5$ days duration. Early administration of IVIG reduces the frequency of CAL in patients with $\mathrm{KD}$.

\section{FINE NEEDLE ASPIRATION CYTOPATHOLOGY OF PEDIATRIC LYMPHADENOPATHY}

doi:10.1136/archdischild-2012-302724.0853

HG Ahmed. Pathology, College of Medicine, University of Hail, Hail, Saudi Arabia

Background Pediatric lymphadenopathy is a challenging medical situation for the patient, the parent, and the physician. Although the bulk of these masses will be benign the fear of malignancy is omnipresent. Therefore, the objective of this study was to identify the common causes of lymphadenopathy among Sudanese children.

Methods One hundred pediatric patients presenting with peripheral lymphadenopathy were included in the study, their ages ranging from 2 to 14 years, with a mean age of 7 years. Demographic characteristics, clinical manifestations and FNA materials were prospectively obtained.

Results FNA was performed under general anesthesia in 100 cases $(100 \%)$. There were no technical complications. All cases confirmed adequacy of specimen. Overall, FNA demonstrated 90(90\%) benign lesions and 10(10\%) malignant diagnosis. The benign lesion was reactive lymphoid hyperplasia $(n=64)$, followed by benign granulomatous disease $(n=26)$. Of the 10 cases diagnosed with malignancy, $7(70 \%)$ were cases of non-Hodgkin's lymphoma and the remaining $3(3 \%)$ were Hodgkin's lymphoma cases.

Conclusion lymphoblastic leukemia can be suspected in pediatric patients with lymphadenopathy. FNA provides a useful tool in the diagnosis of pediatric patients with peripheral lymphadenopathy.

\section{4}

A RARE, BUT SERIOUS SIDE EFFECT OF METHYLPHENIDATE

doi:10.1136/archdischild-2012-302724.0854

1,2S Damodaran, ${ }^{3} \mathrm{G}$ Erumbala, ${ }^{4} \mathrm{D}$ Abraham, 'L Doddamani. 'Paediatrics, James Paget Hospital, Great Yarmouth: 2PICU, Addenbrookes Hospital, Cambridge: ${ }^{3}$ Paediatrics, Milton Keynes General Hospital NHS Foundation Trust, Milton Keynes; ${ }^{4}$ Neonatal Unit, Birmingham Women's Hospital, Birmingham, UK

Introduction Anaemia, leucopenia and thrombocytopenia have been rarely reported in patients receiving methylphenidate. There is no recommendation for routine blood testing unless clinically indicated. We report two children who developed reversible neutropenia on treatment with methylphenidate.

Method-case reports Case1: Routine blood test in a 14 year old boy with $\mathrm{ADHD}$ on Concerta XL 54 mg daily showed significant neutropenia (white cell count (WCC) - 3.1, neutrophil count - 0.8). The drug was discontinued following which WCC and neutrophil counts increased. Concerta was restarted but count started to decline after 2 weeks. Hence the drug was stopped. Since then, his counts have remained normal.

Case 2: Routine blood test in a 12 year old boy with ADHD on concerta XL $36 \mathrm{mg}$ and $10 \mathrm{mg}$ of methylphenidate immediate release daily showed neutropenia (1.72). The count was further reduced to 1.55 after a month. There was no history of any viral infection during or immediately before this period. Neutrophil count normalised 1 month after stopping medication.

Discussion Methylphenidate is the most commonly prescribed stimulant for $\mathrm{ADHD}$. There are wide variation in haematological 